\title{
BMJ Open Rapid increase of gonorrhoea cases in Guangdong Province, China, 2014-2017: a review of surveillance data
}

\author{
Cheng Wang (D) , ${ }^{1,2}$ Weiming Tang (D) , 2,3 Peizhen Zhao, ${ }^{1,2}$ Joseph Tucker, ${ }^{3}$ \\ Lei Chen, ${ }^{1,2}$ M Kumi Smith (D) , ${ }^{4}$ Ngai Sze Wong, ${ }^{5}$ Willa Dong, ${ }^{6}$ Bin Yang, ${ }^{1,2}$ \\ Heping Zheng ${ }^{1,2}$
}

To cite: Wang C, Tang W, Zhao $\mathrm{P}$, et al. Rapid increase of gonorrhoea cases in Guangdong Province, China, 2014-2017: a review of surveillance data. BMJ Open 2019:9:e031578. doi:10.1136/ bmjopen-2019-031578

- Prepublication history and additional material for this paper are available online. To view these files, please visit the journal online (http://dx.doi. org/10.1136/bmjopen-2019031578).

Received 13 May 2019

Revised 26 August 2019

Accepted 07 October 2019

Check for updates

(c) Author(s) (or their employer(s)) 2019. Re-use permitted under CC BY-NC. No commercial re-use. See rights and permissions. Published by BMJ.

For numbered affiliations see end of article.

Correspondence to

Dr Heping Zheng;

zhhpf@hotmail.com

\section{ABSTRACT}

Objectives An increased trend in the number of reported gonorrhoea cases has been observed between 2014 and 2017 in China. This study aims to describe the reported epidemic of gonorrhoea and potential driving forces in Guangdong Province, China.

Design A review of surveillance data.

Participants Three different sources of data from Guangdong Province were analysed: gonorrhoea cases reported to the Chinese sexually transmitted infections (STI) case report system (CRS); a clinic-based retrospective study conducted to collect information on annual gonorrhoea screening coverage and data from the Guangdong governmental sentinel surveillance network (SSN) to examine the gonorrhoea prevalence among males attending STI clinics.

Outcome measures Reported incidence of gonorrhoea, number of reported gonorrhoea cases, number of screening tests for gonorrhoea and gonorrhoea prevalence. Results The STI CRS data showed that the reported incidence of gonorrhoea has increased rapidly from 15.7 cases per 100000 population in 2014 to 27.3 cases per 100000 in 2017 in Guangdong $(p<0.001)$. Regions with a reported incidence of gonorrhoea cases of more than 10 cases per 100000 expanded from 7 cities in 2014 to 13 cities in 2017. The SSN data showed that the gonorrhoea prevalence among males attending STI clinics increased from $2.7 \%$ in 2015 to $3.6 \%$ in 2017 ( $p=0.14$ ). The retrospective study showed that the increased rate of screening for gonorrhoea between 2014 and 2017 was $35.0 \%$, which was much lower than the increased rate of the number of reported gonorrhoea cases (123.3\%). Conclusions The number of gonococcal infections is rapidly rising in Guangdong, China. Expanded screening coverage, use of more sensitive diagnostics and increase of gonorrhoea prevalence are three potential contributors to the epidemic. Additional targeted intervention strategies are necessary in the future to control the epidemic.

\section{INTRODUCTION}

Gonorrhoea continues to be a major public health concern in many settings because of the high incidence and antimicrobial resistance. ${ }^{1-3}$ The WHO estimated that in 2012, there were 78 million cases among adults aged 15-49 years worldwide with a global incidence

\section{Strengths and limitations of this study}

This is one of very limited studies analysing potential driving forces of an increase trend in the number of reported gonorrhoea cases between 2014 and 2017 in China

- Our study examined the multiple aspects of the gonorrhoea epidemic by analysing data from three different sources.

- The number of reported gonorrhoea cases might be underestimated based on a large number of private clinics that do not report gonorrhoea cases in China.

- This study was conducted in Guangdong, one of the most economically developed provinces in China; therefore, generalisability is a limitation of this study.

rate of 19 per 1000 females and 24 per 1000 males. ${ }^{4}$ Gonorrhoea infection is a leading cause of infertility, pelvic inflammatory disease and ectopic pregnancy. Additionally, gonorrhoea is known to increase transmission and acquisition of HIV by inducing the production of cytokines, chemokines and defensins to modulate HIV infection and infectivity. ${ }^{15-7}$

The number of reported gonorrhoea cases gradually declined in China from 2000 to 2013, based on the Chinese sexually transmitted infections (STI) case report system (CRS) ${ }^{8}$ This official STI CRS is a vital reference for STI control policy making, although repeated or erroneous reporting cases exist. However, there has been a recent reversal of this trend with a nearly $30 \%$ increase, from 7.2 cases per 100000 in 2014 to 10.1 cases per 100000 in 2017..$^{9}$ An increasing trend was also observed in several other countries, including the $\mathrm{USA},{ }^{10}$ the $\mathrm{UK},{ }^{11}$ France, ${ }^{12}$ Australia and Greenland. ${ }^{13}$ Multiple factors including greater screening coverage, use of more sensitive diagnostics and high levels of unsafe sexual behaviour among certain subpopulations were likely contributors. ${ }^{14-16}$ 
However, there has been limited analysis of this trend in China to confirm the increase and the driving forces to date, which is critical to informing the design of tailored intervention programme.

To address this gap, we conducted this study in Guangdong Province. We selected this province because of a high burden of gonorrhoea, ${ }^{9}$ and a strong case reporting system. ${ }^{17}$ In 2017, the total burden of gonorrhoea in China was 10.1 cases per 100000 population with Guangdong province reporting 27.3 cases per 100000 population. ${ }^{9}$ Guangdong has consistently ranked among the top three provinces with a high incidence of reported gonorrhoea cases and ranking first on the number of newly reported gonorrhoea cases in China in the last decade. ${ }^{89}$ Additionally, Guangdong has the most complete and effective surveillance system for STI among all the provinces in China ${ }^{17}$ and is able to provide high-quality surveillance data.

This study aims to describe the reported epidemic of gonorrhoea in Guangdong, China, and potential driving forces of the increasing.

\section{METHODS}

To investigate the reasons for the increasing gonorrhoea epidemic in Guangdong Province, we analysed data from three sources. First, we examined data from 2004 to 2017 from the Guangdong Provincial STI CRS to describe the gonorrhoea epidemic. Second, we conducted a clinicbased retrospective study to explore the role of expanding screening coverage on the increasing trend for gonorrhoea between 2014 and 2017. Third, we analysed the data from the Guangdong governmental sentinel surveillance network to determine whether gonorrhoea prevalence among males attending STI clinics increased between 2014 and 2017. The detailed information for these three different data sources is described below (online supplementary figure 1 ).

\section{Guangdong provincial STI CRS}

The STI CRS was initiated by the Chinese Center for Disease Control and Prevention in 1985, and aims to survey syphilis, gonorrhoea, Chlamydia trachomatis, Condyloma acuminata and genital herpes across the country. The Guangdong Provincial STI CRS comprises 3261 governmental health facilities within the 21 municipalities of Guangdong Province. In all health facilities (which include STI clinics, designated dermatovenereology centres, gynaecology and genitourinary clinics, maternity and children's hospitals and other healthcare entities) covered by this system, newly diagnosed STI cases are required to be reported to the STI CRS by each facility. Doctors or their assistants complete a standardised STI reporting card with socio-demographic (identification number, date of birth, sex, marital status, address and occupation), clinical (diagnostic evidence, and the onset of symptoms, diagnosis and death) and epidemiological information (transmission route). As of
2005, STI case-reporting transitioned from paper reports to electronic online submissions in China. According to national guidelines, ${ }^{18}$ gonorrhoea cases are defined by positive diagnostic tests of sputum smear, culture or nucleic acid; history of sexual risk and characteristic clinical manifestations. We extracted data between 2004 and 2017 from the Guangdong Provincial STI CRS in this study.

\section{Clinic-based retrospective study}

A clinic-based retrospective study was conducted to collect information from January 2014 to December 2017 on annual gonorrhoea screening coverage, the proportion of different types of gonorrhoea testing methods including smear, culture, PCR and immunofluorescence, and the proportion of gonorrhoea cases detected by the above four testing methods. We selected hospitals for this study according to the following procedures. First, we extracted data from the Guangdong provincial STI CRS on the number of reported gonorrhoea cases of each city in Guangdong from 2014 to 2017. The cities with a higher rate of increase in the number of reported gonorrhoea cases between 2014 and 2017 than that of Guangdong province as a whole were selected for this study. Finally, within each city, the hospitals with a higher rate of increase in reported gonorrhoea cases between 2014 and 2017 than that of the city were then selected. A total of 10 cities (Shenzhen, Guangzhou, Foshan, Zhuhai, Zhongshan, Jiangmen, Dongguan, Huizhou, Jieyang and Meizhou) and 40 public hospitals were finally selected in the study. Trained interviewers contacted the hospitals and completed a questionnaire by transcribing all existing medical records with the assistance of staff members from Information section.

\section{Guangdong governmental sentinel surveillance network}

To increase the accuracy and reliability with which gonorrhoea incidence could be assessed, a governmental sentinel surveillance network comprising 10 STI clinics in 10 cities (Zhuhai, Dongguan, Foshan, Jiangmen, Qingyuan, Shaoguan, Jieyang, Shantou, Maoming and Zhaoqing) with a high burden of gonorrhoea was established in 2015 (online supplementary figure 2). A serial cross-sectional survey was routinely conducted in these sites among males attending STI clinics aged at least 15 years old from April to June of each year. A standardised survey protocol designed by the Guangdong Provincial Centre for STI Control was sent to these sites for data collection and management. A minimum sample size of 200-250 participants was required for each site. ${ }^{16}$ After providing verbal informed consent, those who agreed to participate in the study were referred to a separate, quiet room to complete a questionnaire including demographic, behavioural and clinical information with the help of a research assistant. Urine samples were collected from all eligible participants for gonorrhoea testing by nucleic acid amplification tests. 


\section{Statistical analysis}

Measures derived from the Guangdong provincial STI CRS data included: (1) the reported incidence of gonorrhoea which was calculated by dividing the number of newly diagnosed gonorrhoea cases by the total population of Guangdong province in a specified year; (2) the average yearly rate of increase in incidence which was calculated as a geometric mean by the use of the following formula: (incidence at years $\mathrm{n}$ divided by incidence at years $m)^{\wedge}(1 /[n-m])-1$. Guangdong provincial population data were obtained from the Guangdong Bureau of Statistics of China. ${ }^{19}$

Measures for the clinic-based retrospective study included: (1) the average yearly rate of increase in the number of screening tests for gonorrhoea (see above); (2) the increased rate of screening amount for gonorrhoea between 2014 and 2017 which was calculated by the following formula: ([screening amount during year 2017screening amount during year 2014]/screening amount during year 2014)*100\% and (3) the rate of increase in the number of reported gonorrhoea cases between 2014 and 2017 (see above).

We calculated gonorrhoea prevalence from the Guangdong governmental sentinel surveillance network data, which refers to the total number of gonorrhoea cases divided by the total population of Guangdong province in a specified year.

We used the $\chi^{2}$ trend test to assess trends over years. Multivariable logistic regression was applied to examine the factors associated with gonorrhoea infection among males attending STI clinics between 2015 and 2017.

All analyses were conducted using SAS V.9.4 statistical software .

\section{Patient and public involvement}

The participants were not involved in the development of the research question, outcome measures, design, recruitment and conduct of this study.
RESULTS

\section{Data from Guangdong provincial STI CRS}

A total of 85671 gonorrhoea cases were reported in Guangdong Province between 2014 and 2017. The maleto-female ratio of gonorrhoea cases decreased from 6.6 in 2014 to 6.5 in $2017\left(\chi^{2}=607.11, \mathrm{p}<0.001\right)$. While incidence decreased from 2004 to 2014, the reported incidence of gonorrhoea grew rapidly with an average yearly increase of $20.2 \%$ from 15.7 cases per 100000 in 2014 to 27.3 cases per 100000 in $2017\left(\chi^{2}=4133.81, \mathrm{p}<0.001\right)$, which is similar to the trend in reporting incidence of male gonorrhoea cases (figure 1). In 2014, only seven cities located in the Pearl River Delta (Shenzhen, Foshan, Zhuhai, Zhongshan, Guangzhou, Dongguan and Jiangmen) had a reported incidence of gonorrhoea over 10 cases per 100000 . In 2017, this epidemic was extended to an additional city in the Pearl River Delta (Huizhou), as well as four cities in the northern Guangdong (Qingyuan, Yunfu, Shaoguan and Heyuan), and one city in the eastern region (Shantou) (online supplementary figure 3).

\section{Data from a clinic-based retrospective study}

The clinic-based retrospective study showed that the number of screening tests for gonorrhoea increased from 155747 in 2014 to 210238 in $2017(\mathrm{p}<0.001)$, with an average yearly increase of $10.5 \%$. Among the five different testing methods for gonorrhoea, the number of PCR tests grew most rapidly with an average yearly increase of $39.9 \%$ from $10792(6.9 \%)$ in 2014 to $29543(14.1 \%)$ in $2017(\mathrm{p}<0.001)$, followed by culture testing with an average yearly increase of $13.5 \%$ from $42172(27.1 \%)$ in 2014 to $61675(29.3 \%)$ in $2017(\mathrm{p}<0.001)$. Overall, the smear test was the most frequently used among the five different testing methods between 2014 and 2017.

The proportion of gonorrhoea cases detected by PCR increased from $11.3 \%$ to $12.9 \%$ between 2014 and 2017 $(\mathrm{p}=0.57)$. This was similar to the immunofluorescence test, which increased from $1.5 \%$ to $4.5 \%(\mathrm{p}<0.001)$. By

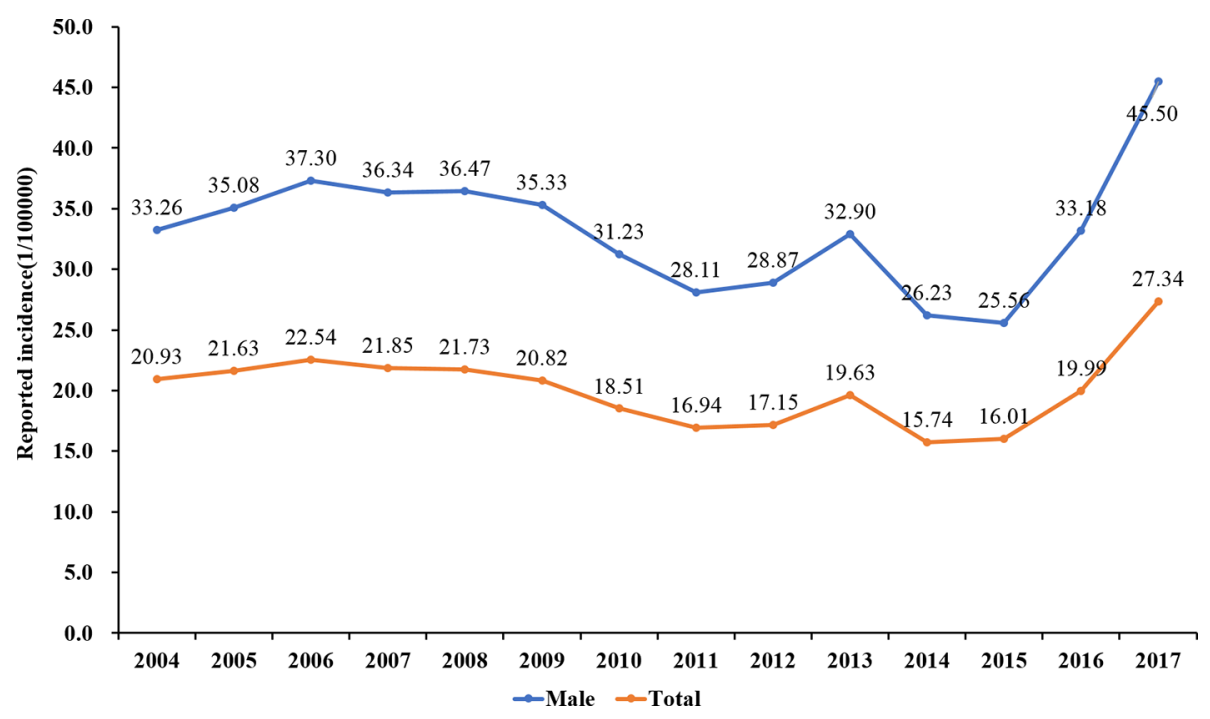

Figure 1 Reported incidence of gonorrhoea from 2004 to 2017 based on the sexually transmitted infections case report system. 
Table 1 Screening amount and positive gonorrhoea cases detected in the 40 hospitals, 10 cities from 2014 to 2017, a retrospective study

\begin{tabular}{|c|c|c|c|c|c|}
\hline Test method & 2014 & 2015 & 2016 & 2017 & $\chi^{2}(\mathbf{p})$ \\
\hline \multicolumn{6}{|l|}{ Smear* } \\
\hline No. of tests (\%) & $88879(57.1)$ & $76202(44.4)$ & $74784(40.8)$ & $97774(46.5)$ & $3607.0(<0.001)$ \\
\hline No. of positive cases (\%) & $2389(48.0)$ & $2546(46.1)$ & $3185(42.4)$ & $5005(44.6)$ & $824.2(<0.001)$ \\
\hline \multicolumn{6}{|l|}{ Culture* } \\
\hline No. of tests (\%) & $42172(27.1)$ & $67137(39.2)$ & 72031 (39.3) & $61675(29.3)$ & $22.5(<0.001)$ \\
\hline No. of positive cases (\%) & $1948(39.2)$ & $2339(42.3)$ & $3262(43.5)$ & $4267(38.0)$ & $470.5(<0.001)$ \\
\hline \multicolumn{6}{|l|}{$\mathrm{PCR}^{*}$} \\
\hline No. of tests (\%) & $10792(6.9)$ & $12568(7.3)$ & $18235(9.9)$ & $29543(14.1)$ & $6203.5(<0.001)$ \\
\hline No. of positive cases (\%) & $564(11.3)$ & $573(10.4)$ & $818(10.9)$ & $1445(12.9)$ & $0.3(0.57)$ \\
\hline \multicolumn{6}{|l|}{ Immunofluorescence* } \\
\hline No. of tests (\%) & $13904(8.9)$ & $15549(9.1)$ & $18384(10.0)$ & $21246(10.1)$ & $270.2(<0.001)$ \\
\hline No. of positive cases (\%) & $74(1.5)$ & $70(1.3)$ & 239 (3.2) & $503(4.5)$ & $299.2(<0.001)$ \\
\hline \multicolumn{6}{|l|}{ Total $^{\star}$} \\
\hline $\begin{array}{l}\text { No. of tests (\%, increased } \\
\text { rate compared with 2014) }\end{array}$ & 155747 & $171456(10.1)$ & $183434(17.8)$ & $210238(35.0)$ & $4525.4(<0.001)$ \\
\hline No. of positive cases & 4975 & 5528 & 7504 & 11220 & $1318.1(<0.001)$ \\
\hline $\begin{array}{l}\text { No. of reported cases }(\%, \\
\text { increased rate compared with } \\
2014) \dagger\end{array}$ & 3600 & $3910(8.6)$ & $5627(56.3)$ & 8040 (123.3) & $4133.8(<0.001)$ \\
\hline
\end{tabular}

*These data for the 10 cities were obtained from the retrospective study.

†These data for the 10 cities were from STI CRS.

contrast, the proportion of gonorrhoea cases detected by the smear declined from $48.0 \%$ in 2014 to $44.6 \%$ in 2017 $(\mathrm{p}<0.001)$, and by the culture declined from $39.2 \%$ in 2014 to $38.0 \%$ in $2017(\mathrm{p}<0.001)$.

The rate of increase in the number of screening tests for gonorrhoea during the period from 2014 to 2017 was $35.0 \%$, which was much lower than the increased rate of the number of reported gonorrhoea cases $(123.3 \%)$ during that time period (table 1 and figure 2).

Data from the Guangdong governmental sentinel surveillance network

Under the sentinel surveillance network programme, a total of 5440 participants were recruited, interviewed and

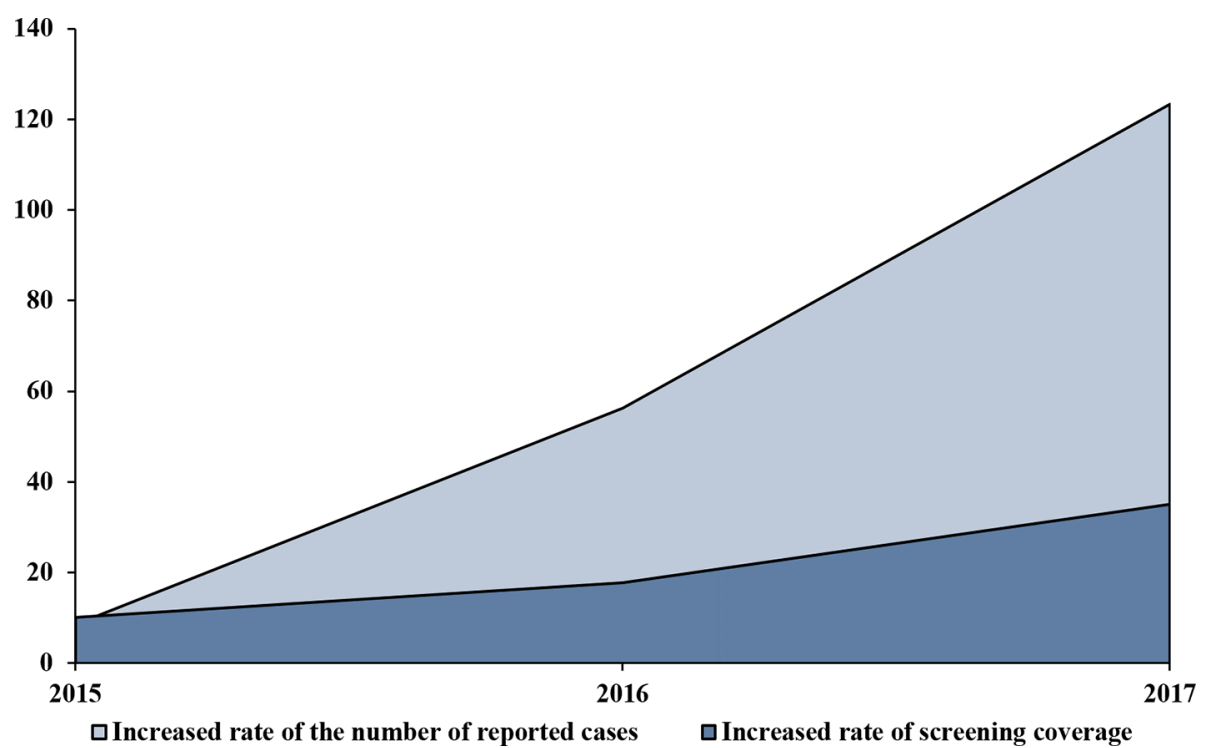

Figure 2 The yearly increased rate of screening coverage from the retrospective study and the number of reported cases from the sexually transmitted infections case report system based on 2014 in the 40 hospitals, 10 cities. 
Table 2 Sexual risk behaviour among male attending sexually transmitted infections (STI) clinics between 2015 and 2017

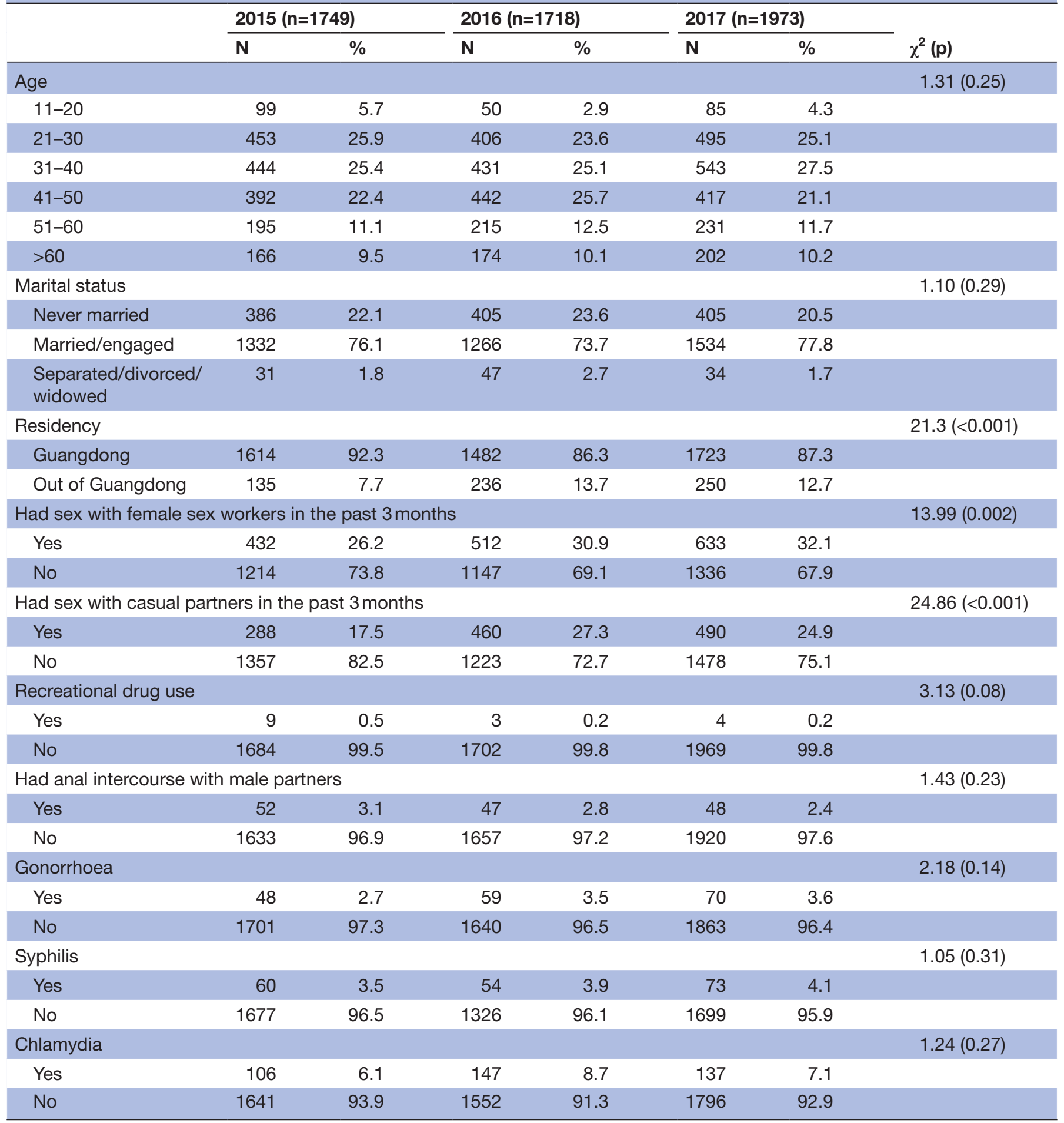

tested between 2015 and 2017. The gonorrhoea prevalence among males attending STI clinics increased with a yearly average of $14.94 \%$, from $2.7 \%$ in 2015 to $3.6 \%$ in 2017, although this increase was not significant $(p=0.14)$. The proportion of male STI clinic attendees reporting having sex with female sex workers in the past 3 months grew significantly from $26.2 \%$ in 2015 to $32.1 \%$ in 2017 $(p=0.002)$. The same trend was also observed for the proportion of participants having sex with casual partners in the past 3 months from $17.5 \%$ in 2015 to $24.9 \%$ in 2017 $(\mathrm{p}<0.001)$ (table 2$)$.

In the multivariable model adjusted for age, marital status and residency, two factors were positively associated with gonorrhoea infection: having sex with female sex workers in the past 3 months (aOR: $2.55,95 \% \mathrm{CI}$ : 1.89 to 3.45 ) and having sex with casual partners in the past 3 months (aOR: $1.49,95 \% \mathrm{CI}: 1.08$ to 2.07 ) (table 3). 
Table 3 Factors associated with gonorrhoea infection among males attending clinics between 2015 and 2017

\begin{tabular}{|c|c|c|c|}
\hline \multirow[b]{2}{*}{ Characteristics } & \multirow[b]{2}{*}{ n (\%) } & \multirow{2}{*}{$\begin{array}{l}\text { Crude model } \\
\text { OR }(95 \% \mathrm{Cl})\end{array}$} & \multirow{2}{*}{$\begin{array}{l}\text { Adjusted model† } \\
\text { OR }(95 \% \mathrm{Cl})\end{array}$} \\
\hline & & & \\
\hline \multicolumn{4}{|c|}{ Had sex with female sex workers in the past 3 months } \\
\hline No & $3661(69.8)$ & Ref. & Ref. \\
\hline \multicolumn{4}{|c|}{ Had sex with casual partners in the past 3 months } \\
\hline No & $4022(76.8)$ & Ref. & Ref. \\
\hline \multicolumn{4}{|c|}{ Recreational drug use } \\
\hline Yes & $16(0.3)$ & $1.94(0.26$ to 14.76$)$ & $1.73(0.23$ to 13.26$)$ \\
\hline No & $5296(99.7)$ & Ref. & Ref. \\
\hline \multicolumn{4}{|c|}{ Had anal intercourse with male partners } \\
\hline
\end{tabular}

${ }^{*} \mathrm{P}<0.01$.

†Adjusted model was adjusted for age (continuous), marital status and residency.

\section{DISCUSSION}

A rapid increase in the number of reported gonorrhoea cases has been observed since 2014 in China. This study applied multiple sources of surveillance data from Guangdong province to identify the reasons. Our data suggest that expanded screening coverage, use of more sensitive diagnostics and increase in gonorrhoea prevalence are three potential contributors to the epidemic. Findings from this study provide insights for the design of tailored gonorrhoea intervention programme in China.

Our study found that the reported incidence of gonorrhoea in Guangdong has been increasing since 2014, and the number of cities with a reporting incidence of gonorrhoea more than 10 cases per 100000 expended from 7 in 2014 to 13 in 2017 across Guangdong Province based on the provincial STI CRS. This increasing epidemic was similar to the epidemic across China, as well as that of many other countries, such as an $18.5 \%$ rise between 2015 and 2016 in the USA, ${ }^{10}$ and a $22 \%$ rise between 2017 and 2016 in the UK. ${ }^{11}$ Data from the Guangdong governmental sentinel surveillance network echoed this increasing trend among males attending STI clinics from 2015 to 2017.

Results from the clinic-based retrospective study indicated that gonorrhoea screening uptake levels have been rising with a yearly increase of $10.52 \%$ from 2014 to 2017, especially with the increased use of PCR which is the most sensitive test for Neisseria gonorrhoea. ${ }^{20}$ Similar increases due to growing PCR use were also observed in several European countries. ${ }^{13} 21$ In China, this increase can be largely attributed to the Zero Mark-up Drug policy launched by the Chinese government in $2009^{22}$ and piloted in all county level public health facilities throughout Guangdong in 2013. ${ }^{23}$ The Zero Mark-up Drug policy mandates that all drugs must be sold with no markup from wholesale to retail price in all public health facilities. ${ }^{24}$ According to our previous qualitative study with physicians, ${ }^{25}$ this policy led to changes in diagnostic behaviours of doctors for gonorrhoea, such as increasing reliance on laboratory analysis over syndromic approaches. ${ }^{26}$ For example, prior to the implementation of this policy, physicians reported typically diagnosing male patients with gonococcal urethritis based on symptoms without conducting laboratory testing. ${ }^{27}$ After the policy was launched, physicians chose to provide laboratory testing to all male patients with urethral discharge for the diagnosis. This policy likely caused a rapid increase in the number of screening tests conducted for gonorrhoea in public health facilities.

While the screening coverage for gonorrhoea increased considerably between 2014 and 2017, we found that the rate of increase in gonorrhoea screening was much lower than the rate of increase in the number of reported gonorrhoea cases. Additionally, data from the governmental sentinel surveillance network indicated that the proportion of males attending STI clinics engaging in risky sexual behaviour has also grown rapidly since 2015, which is an important factor relevant to the spread of STI. ${ }^{28}$ These results suggest that there was an increase in the population prevalence of gonorrhoea even if screening levels were not considered.

Our study has several limitations. First, only public health facilities are mandated to report STI in Guangdong Province. The number of reported gonorrhoea cases is likely underestimated based on a large number of private clinics that do not report gonorrhoea cases to the STI CRS. ${ }^{29}$ Second, the Guangdong sentinel surveillance network only surveys males attending STI clinics, but not female attendees. Nevertheless, the trend of the incidence rate of male gonorrhoea cases can be used to 
represent gonorrhoea cases overall in Guangdong. Third, there may have selection bias induced by non-response for the survey of sentinel surveillance network. Fourth, this study was conducted in Guangdong, one of the most economically developed provinces in China. We are uncertain whether the results can be generalised to other province in China. However, this study provides insights on contributing factors to the gonorrhoea epidemic and for gonorrhoea prevention at the provincial level.

\section{CONCLUSIONS}

In conclusion, the number of gonococcal infections is rapidly rising in Guangdong, China. We used data from multiple sources to explore reasons for this increase. Expended screening coverage, use of more sensitive diagnostics and increase of gonorrhoea prevalence are three potential contributors to the epidemic. Considering this increase in the number of gonorrhoea cases between 2014 and 2017 coincided with decreasing susceptibility of $\mathrm{N}$ gonorrhoea to currently available antimicrobial drugs ${ }^{30-34}$ prevention of gonorrhoea remains a major public health priority in China. More targeted intervention strategies and efforts, such as early detection and screening strategies, partner management, development of new diagnostics and novel treatment options, should be paid more attention in the future.

\section{Author affiliations}

${ }^{1}$ STD Control, Dermatology Hospital of Southern Medical University, Guangzhou, China

${ }^{2}$ STD Control, Guangdong Provincial Center for Skin Diseases and STIs control, Guangzhou, China

${ }^{3}$ Project-China, University of North Carolina, Guangzhou, China

${ }^{4}$ School of Public Health, University of Minnesota Twin Cities, Minneapolis, Minnesota, USA

${ }^{5}$ Stanley Ho Center for Emerging Infectious Diseases, The Chinese University of Hong Kong, Hong Kong, China

${ }^{6}$ University of North Carolina at Chapel Hill Gillings School of Global Public Health, Chapel Hill, North Carolina, USA

Acknowledgements We thank all the study participants in the 21 city-level STI control centers in Guangdong Province who contributed to this study.

Contributors CW participated in all stages and wrote the manuscript. $\mathrm{HZ}$ designed this study, PZ and LC conducted the field study and analysed the data. WT, JT, MKS, NSW, WD and BY reviewed the manuscript.

Funding This publication is funded by the Dermatology Hospital of Southern Medical University.

Map disclaimer The depiction of boundaries on the map(s) in this article does not imply the expression of any opinion whatsoever on the part of BMJ (or any member of its group) concerning the legal status of any country, territory, jurisdiction or area or of its authorities. The map(s) are provided without any warranty of any kind, either express or implied.

Competing interests None declared.

Patient consent for publication Not required.

Ethics approval The study protocol was approved by the institutional review boards of the Dermatology Hospital of Southern Medical University (GDDHLS-20181204).

Provenance and peer review Not commissioned; externally peer reviewed.

Data availability statement Data are available upon reasonable request.
Open access This is an open access article distributed in accordance with the Creative Commons Attribution Non Commercial (CC BY-NC 4.0) license, which permits others to distribute, remix, adapt, build upon this work non-commercially, and license their derivative works on different terms, provided the original work is properly cited, appropriate credit is given, any changes made indicated, and the use is non-commercial. See: http://creativecommons.org/licenses/by-nc/4.0/.

ORCID iDs

Cheng Wang http://orcid.org/0000-0001-8000-9969

Weiming Tang http://orcid.org/0000-0002-9026-707X

M Kumi Smith http://orcid.org/0000-0001-5861-8100

\section{REFERENCES}

1 Awuor C, Bala M, Cole M. Global action plan: to control the spread and impact of antimicrobial resistance to Neisseria gonorrhoeae. World Health Organization, Department of Reproductive Health and Research, 2012.

2 Vos T, Barber RM, Bell B, et al. Global, regional, and national incidence, prevalence, and years lived with disability for 301 acute and chronic diseases and injuries in 188 countries, 1990-2013: a systematic analysis for the global burden of disease study 2013. Lancet 2015;386:743-800.

3 Murray CJL, Barber RM, Foreman KJ, et al. Global, regional, and national disability-adjusted life years (DALYs) for 306 diseases and injuries and healthy life expectancy (HALE) for 188 countries, 1990-2013: quantifying the epidemiological transition. Lancet 2015;386:2145-91

4 Newman L, Rowley J, Vander Hoorn S, et al. Global estimates of the prevalence and incidence of four curable sexually transmitted infections in 2012 based on systematic review and global reporting. PLoS One 2015;10:e0143304.

5 Cohen MS, Hoffman IF, Royce RA, et al. Reduction of concentration of HIV-1 in semen after treatment of urethritis: implications for prevention of sexual transmission of HIV-1. AIDSCAP Malawi Research Group. Lancet 1997;349:1868-73.

6 Unemo M, Shafer WM. Antimicrobial resistance in Neisseria gonorrhoeae in the 21st century: past, evolution, and future. Clin Microbiol Rev 2014;27:587-613.

7 Jarvis GA, Chang TL. Modulation of HIV transmission by Neisseria gonorrhoeae: molecular and immunological aspects. Curr HIV Res 2012;10:211-7.

8 Xiangdong G, Xiaoli Y, Ning J, et al. Epidemiological characteristics and trends of gonorrhea in China from 2000 to 2014. C J Dermatol 2015;5:301-6.

9 Heng G. The Chinese STD survelliance. The 9th national dermotology and STD prevention conference 2018, Inner Mongolie, China, 2018.

10 Centers for Disease Control and Prevention. Sexually transmitted disease surveillance 2017, 2016.

11 Public Health England. Sexually transmiited infections and Chlamydia screening in England, 2017, 2018.

12 Ngangro NN, Viriot D, Fournet N. Bacterial sexually transmitted infections in France: recent trends and patients' characteristics in 2015, 2016.

13 Berntsen S, Karlsen APH, Pedersen ML, et al. Gonorrhoea in Greenland, incidence and previous preventive measures: a review to improve future strategies. Int J Circumpolar Health 2017;76:1350092.

14 Van de Laar M, Spiteri G. Increasing trends of gonorrhoea and syphilis and the threat of drug-resistant gonorrhoea in Europe. Euro Surveill 2012;17:20225.

15 Wi T, Lahra MM, Ndowa F, et al. Antimicrobial resistance in Neisseria gonorrhoeae: global surveillance and a call for international collaborative action. PLoS Med 2017;14:e1002344.

16 Qin Q, Tang W, Ge L, et al. Changing trend of HIV, syphilis and hepatitis $\mathrm{C}$ among men who have sex with men in China. Sci Rep 2016;6:31081.

17 Yang L-G, Tucker JD, Wang C, et al. Syphilis test availability and uptake at medical facilities in southern China. Bull World Health Organ 2011;89:798-805.

18 Zheng H, Wang B, Shi Lian QW, et al. National standard of the people's Republic of China: diagnostic criteria for Gonorrhea (GB 20466-2007). Beijing: Standards Press of China, 2007.

19 Guangdong Bureau of Statistics. Guangdong Province yearly statistical data. Available: http://www.gdstats.gov.cn/

20 Budkaew J, Chumworathayi B, Pientong C, et al. Conventional culture versus nucleic acid amplification tests for screening of urethral Neisseria gonorrhea infection among asymptomatic men who have sex with men. Pragmatic and Observational Research 2017;8:167-73. 
21 Bignell C, Unemo M, Radcliffe K, et al. 2012 European guideline on the diagnosis and treatment of gonorrhoea in adults. Int J STD AIDS 2013;24:85-92

22 Liu Y, Lin Z, Ru Y, et al. Small-Scale or full-scale? The zero mark-up drug policy in China. J Interdiscip Math 2017;20:1167-78.

23 Hong J, Chen Q, Li Y. The reforms and development of state - run county -level hospitals after execution of zero - difference policy in drug prices. Modern Hospital 2015;7:97-8.

24 Yi H, Miller G, Zhang L, et al. Intended and unintended consequences of China's zero markup drug policy. Health Aff (Millwood) 2015;34:1391-8.

25 Wang C. Factors associated with the increase of screening amount of gonorrhea: a qualitative study. The Guangdong provincial STD prevention conference, Guangzhou, China, 2019.

$26 \mathrm{He}$ Z, Zhou J. Can zero-markup policy for drug sales in public hospitals resolve the problem of 'seeing a doctor is too expensive' in China? A case study of four municipal general tertiary hospitals at $\mathrm{H}$ City in Z Province. J C Gov 2017;2:329-42.

27 Wang Q, Yang P, Zhong M, et al. Validation of diagnostic algorithms for syndromic management of sexually transmitted diseases. Chin Med J 2003;116:181-6.

28 Homøe A-S, Knudsen A-KS, Nielsen SB, et al. Sexual and reproductive health in Greenland: evaluation of implementing sexual peer-to-peer education in Greenland (the SexInuk project). Int $J$ Circumpolar Health 2015;74:27941.

29 Tucker JD, Yang L-G, Zhu Z-J, et al. Integrated syphilis/HIV screening in China: a qualitative analysis. BMC Health Serv Res 2010;10:58.

30 Fifer $\mathrm{H}$, Natarajan $\mathrm{U}$, Jones $\mathrm{L}$, et al. Failure of dual antimicrobial therapy in treatment of gonorrhea. N Engl J Med 2016;374:2504-6.

31 Cámara J, Serra J, Ayats J, et al. Molecular characterization of two high-level ceftriaxone-resistant Neisseria gonorrhoeae isolates detected in Catalonia, Spain. J Antimicrob Chemother 2012;67:1858-60.

32 Ohnishi M, Golparian D, Shimuta K, et al. Is Neisseria gonorrhoeae initiating a future era of untreatable gonorrhea?: detailed characterization of the first strain with high-level resistance to ceftriaxone. Antimicrob Agents Chemother 2011;55:3538-45.

33 Unemo M, Golparian D, Nicholas R, et al. High-Level cefixime- and ceftriaxone-resistant Neisseria gonorrhoeae in France: novel penA mosaic allele in a successful international clone causes treatment failure. Antimicrob Agents Chemother 2012;56:1273-80.

34 Lahra MM, Enriquez R. Australian meningococcal surveillance programme annual report, 2016. Commun Dis Intell Q Rep 2017;41:E369-82. 